\title{
Comparison of First-Come First-Served and Optimization Based Scheduling Algorithms for Integrated Departure and Arrival Management
}

Bae-Seon Park*, Hyeonwoong Lee, and Hak-Tae Lee / Yeonju Eun and Daekeun Jeon Inha University / Korea Aerospace Research Institute

Zhifan Zhu / Hanbong Lee and Yoon C. Jung Stinger Ghaffarian Technologies / NASA Ames Research Center

AIAA AVIATION 2018

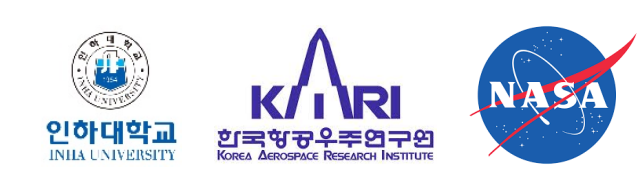




\section{Outline}

$>$ Background

$>$ Two Scheduling Approaches

$>$ Scheduling Result Comparison

$>$ Conclusions 


\section{BACKGROUND}




\section{Background}

$>$ ICN, GMP, and CJU

- Heavy traffic

$>K A R I$ is developing an integrated departure and arrival management system.

- Schedulers (Dep., Arr.)

- Taxi time estimation

- Data management

- Controller display

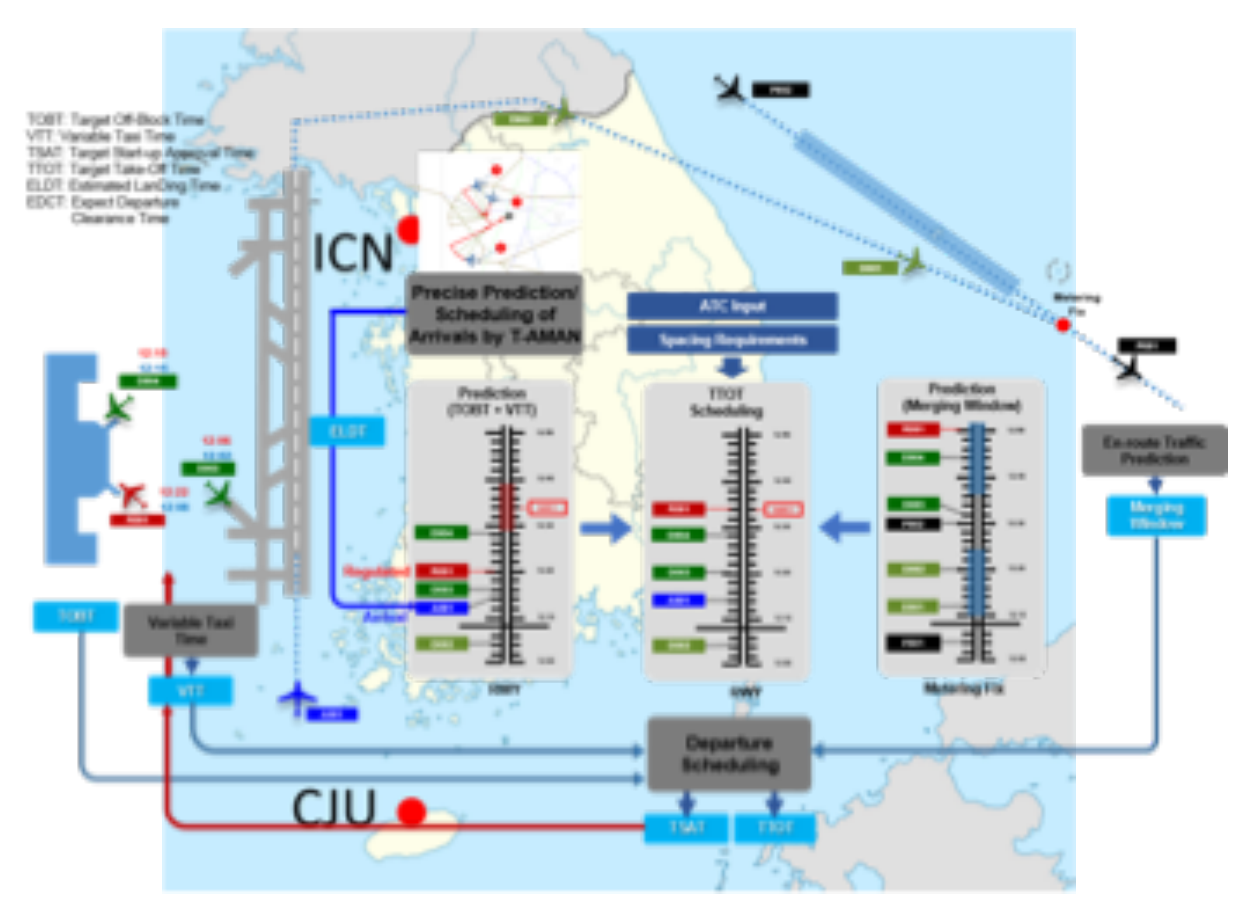




\section{Motivation}

$>$ Scheduling algorithms are one of the key components.

- The Extended First-Come First-Served (EFCFS) scheduler has been developed in Inha University.

- The Mixed Integer Linear Programming (MILP) based scheduler has been developed in KARI in collaboration with NASA.

$>$ Compare two different scheduling algorithms systematically

- Cross verification

- Examine the performance differences between EFCFS and MILP 


\section{TWO SCHEDULING APPROACHES}

Extended First-Come First-Served Approach

Optimization Based Approach

Compatibility of the Two Algorithms

EFCFS Enhancements 


\section{Extended First-Come First-Served Approach*}

$>$ Sequential scheduling based on priority

$>$ Schedule of the higher priority aircraft is frozen first.

$>$ Departure sequence can be switched.

$>$ Minimum delay solution for each flight

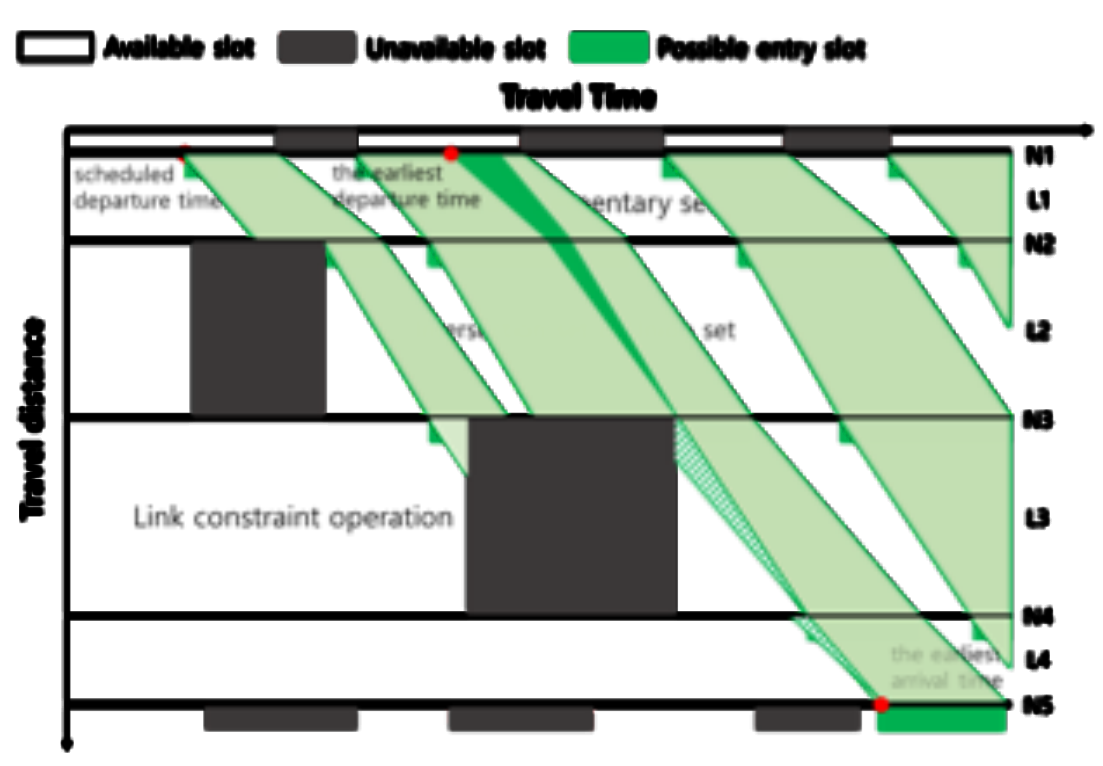

* Park, B., Lee, H., and Lee, H., "Extended First-Come First-Served Scheduler for Airport Surface 


\section{Optimization Based Approach*}

\section{$>$ Based on 3-step approach}

- Scheduling problems of the Step 2 and 3 were formulated as MILP optimization
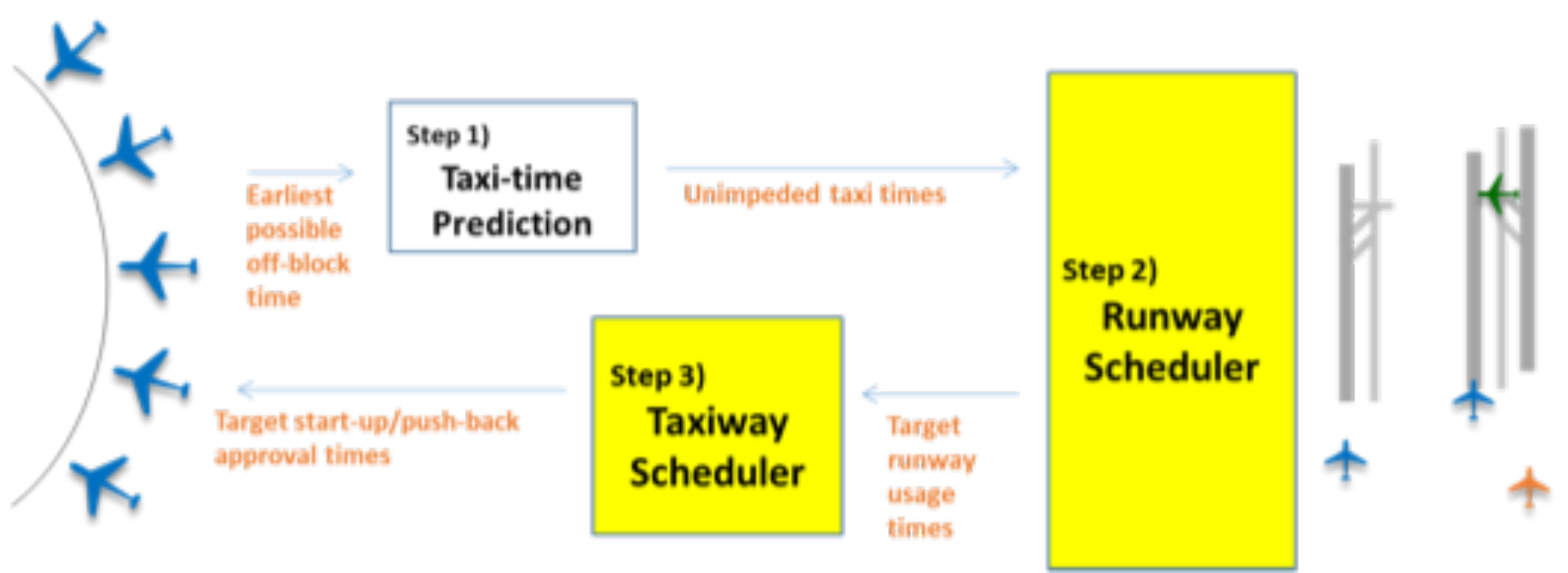

* Eun, Y., Jeon, D., Lee, H., Jung, Y., Zhu, Z., Jeong, M., Kim, H., Oh, E., and Hong, S., “Optimization of 


\section{Optimization Based Approach}

$>$ Runway scheduling

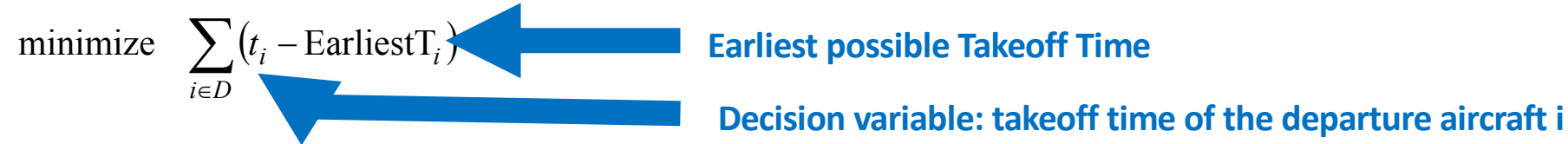

$>$ Taxiway scheduling Decision variable: passage times at all intersections along the taxi routes $\underbrace{\operatorname{minimize} \alpha_{p}\left(\sum_{i \in D, r \in R} \max \left[t_{i, r}-\text { DesiredOffT }_{i, r}, 0\right]\right)}_{\text {Late Take-off Time }}+\alpha_{d}\left(\sum_{i \in D, r \in R} t_{i, r}-\sum_{i \in D, g \in G} t_{i, g}\right)+\alpha_{a}\left(\sum_{i \in A, g \in G} t_{i, g}-\sum_{i \in A, r \in R} t_{i, r}\right)$

$>$ Required separation between aircraft moving on the surface and other considerations about aircraft movements were all formulated as linear equality/inequality constraints. 


\section{Compatibility of the Two Algorithms}

$>$ Use the same predetermined routes

$>$ For arrival flights, taxi scheduling only

- Estimated landing times are given.

$>$ Common constraints

- Earliest possible pushback times of departures

- No deadlock in bi-directional taxiway links

- Aircraft separation along the taxiways

- Runway separation based on aircraft wake turbulence category (WTC)

- Miles-In-Trails at selected fixes (MIT) 


\section{EFCFS Enhancements}

$>$ Runway separation minima based on aircraft WTC*
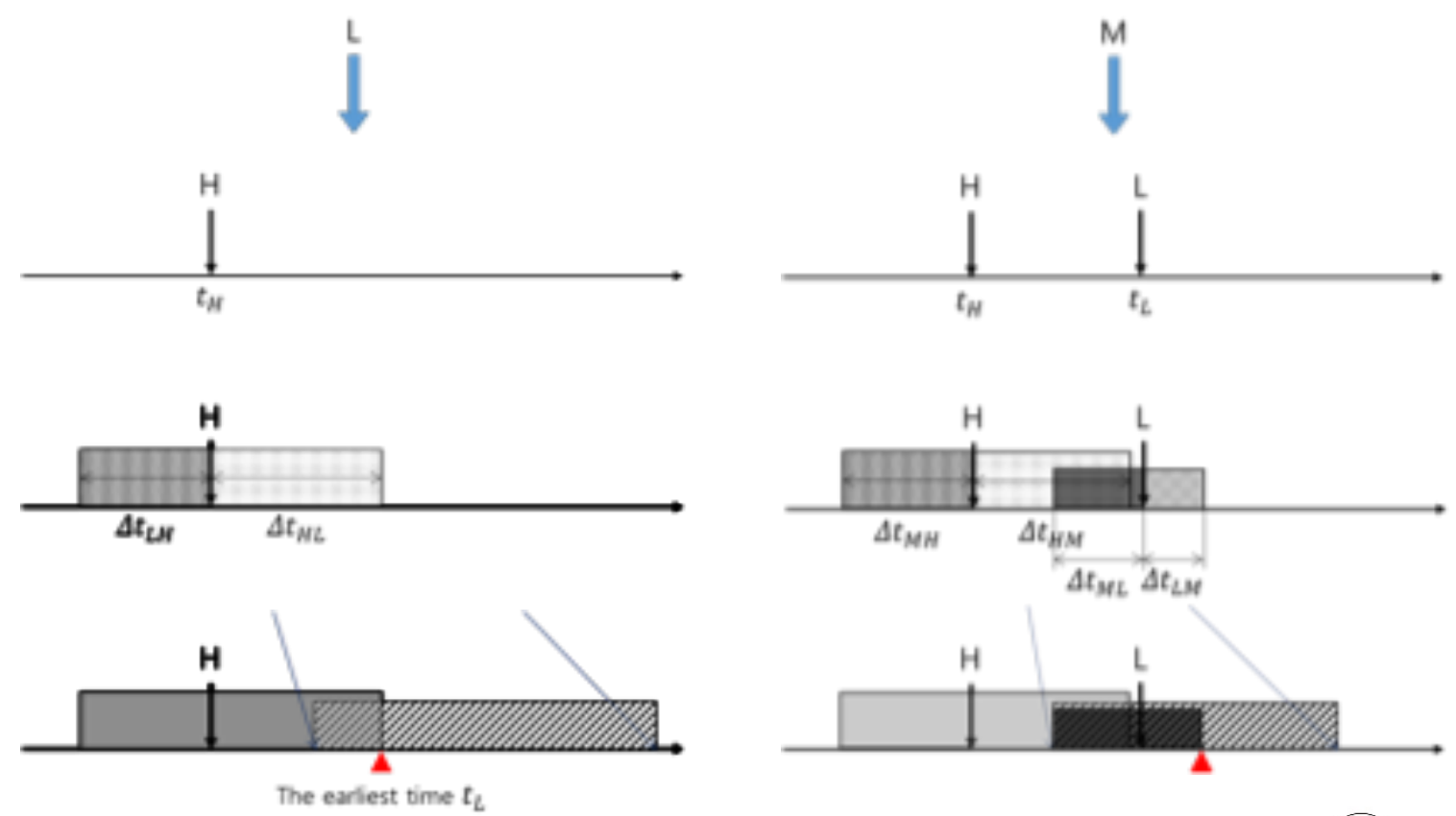

* Park, B., Lee, H., and Lee, H., "Extended First-Come First-Served Scheduler for Airport Surface Operation," International Journal of Aeronautical and Space Sciences (IJASS), Vol.19 (2), 2018. 


\section{EFCFS Enhancements}

\section{A Applying MIT constraints}

- Extending the node-link from the runway to the metering fix

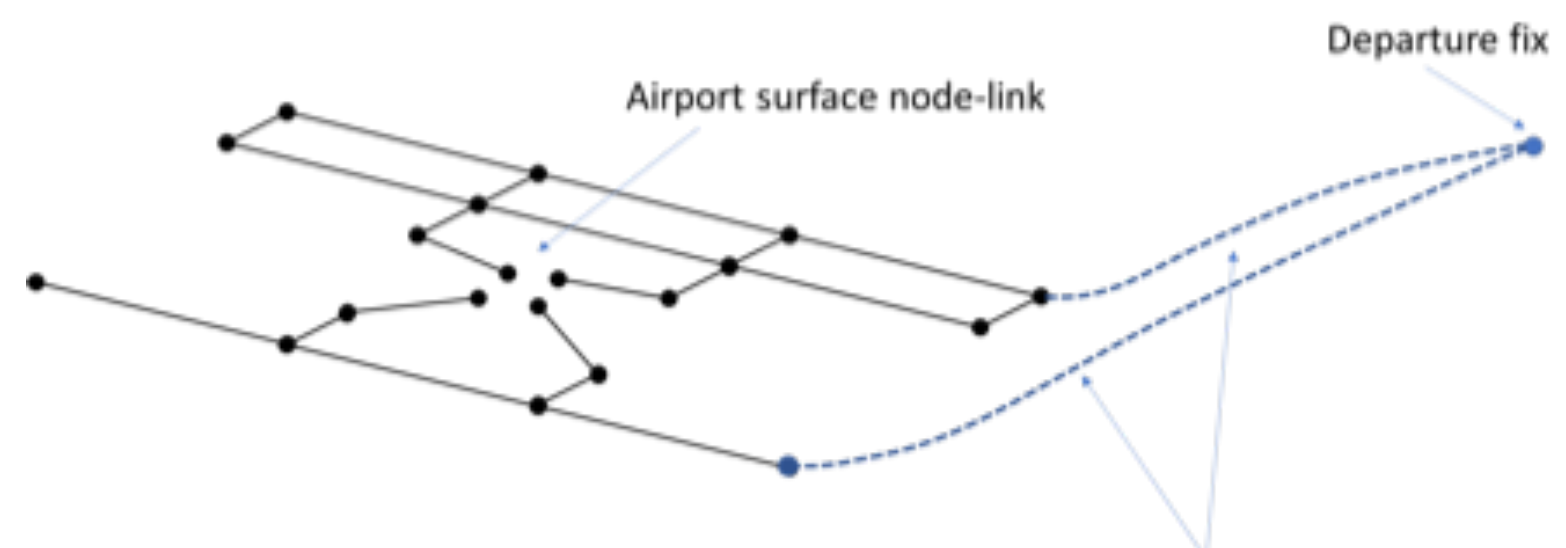

Added links from runways to departure fixes

Extra node-link for departure fix 


\section{SCHEDULING RESULT COMPARISON}

\section{Problem Set}

Scheduling Results

Computation Times 


\section{Incheon International Airport (ICN)}

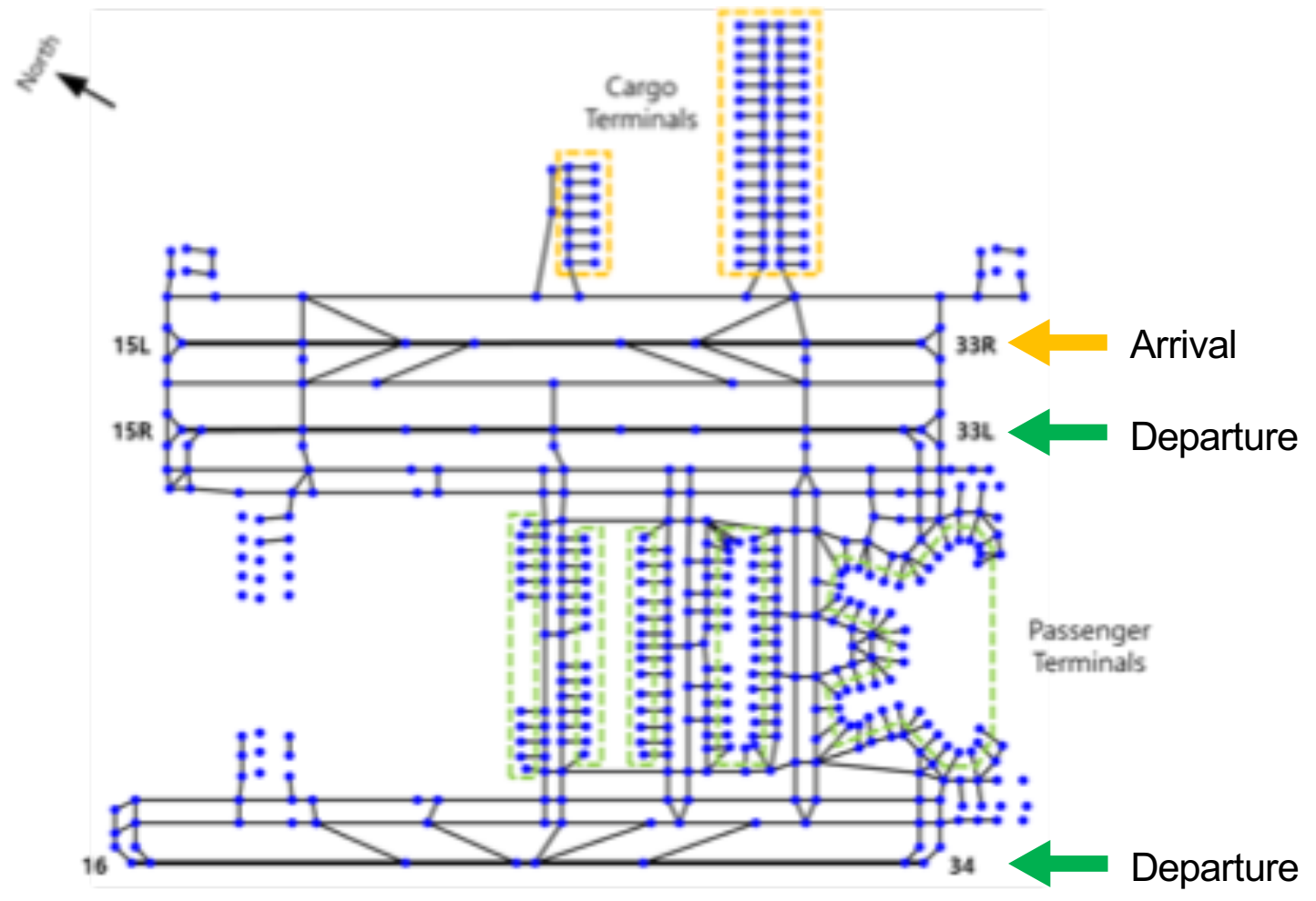




\section{Problem Set}

40 departures and 20 arrivals around 1 hour at ICN

$>$ Fleet mixes of all scenarios are equal

- Departure: 14 Medium and 26 Heavy class aircraft

- Arrival: 7 Medium and 13 Heavy class aircraft

$>$ Arrival landing times were not adjusted

- No landing delays

- Taxi delays can be added while taxiing from runway exits to gates

$>$ Randomly generated 100 scenarios

- Gate departure times, estimated landing times, and gate numbers (Taxi routes) are randomly assigned. 


\section{Problem Set}

$>$ Runways and departure fixes

\begin{tabular}{|c|c|c|c|}
\hline Departure fixes & Runways & \# of flights & MIT \\
\hline \multirow{2nnn}{*}{ East } & \multirow{2}{*}{$15 \mathrm{R} / 33 \mathrm{~L}$} & 5 & $\mathrm{X}$ \\
\cline { 1 - 2 } South East & & 5 & $\mathrm{X}$ \\
\hline \multirow{2}{*}{ South } & $15 \mathrm{R} / 33 \mathrm{~L}$ & 6 & \multirow{2}{*}{0} \\
\cline { 3 - 4 } & $16 / 34$ & 9 & \\
\hline West & $16 / 34$ & 15 & 0 \\
\hline
\end{tabular}

$>$ MIT constraints

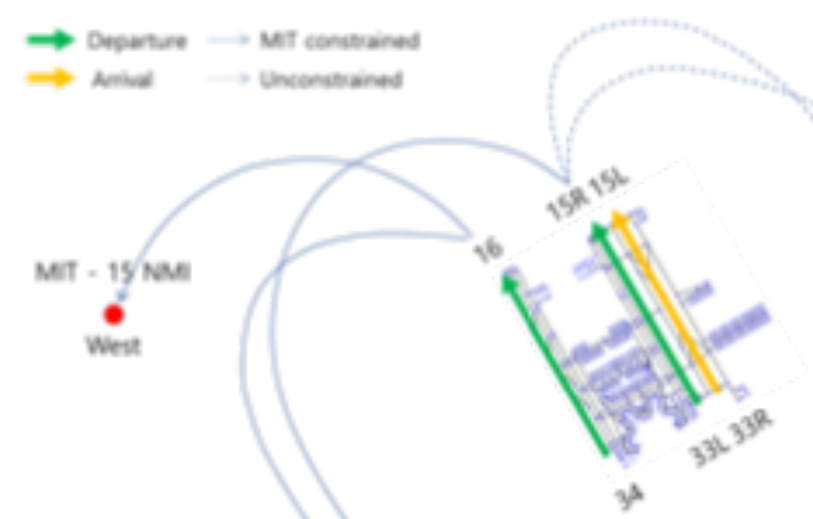

- 15 nautical miles

- Applied to the West and South fixes

- The East and South East fixes were unconstrained 


\section{Scheduling Results}

$>$ Accumulated results for 100 scenarios

$>$ Case 1

- Without MIT constraints (2 mins / 3 mins)

$>$ Case 2

- With MIT constraints (2 mins / 3 mins)

$>$ Case 3

- Artificially increased runway separation minima without MIT constraints for takeoffs ( $2 \rightarrow 5$ mins / $3 \rightarrow 10$ mins) 


\section{* Delay definitions}

\section{Scheduling Results - Case 1}

\section{Delay distributions}

1. Gate delay

= Calculated push-back time - Original push-back time

2. Takeoff delay

= Calculated takeoff time - Original takeoff time

3. Original takeoff time

$=$ Original push-back time + Unimpeded taxi time

- MILP shows smaller average runway takeoff delay

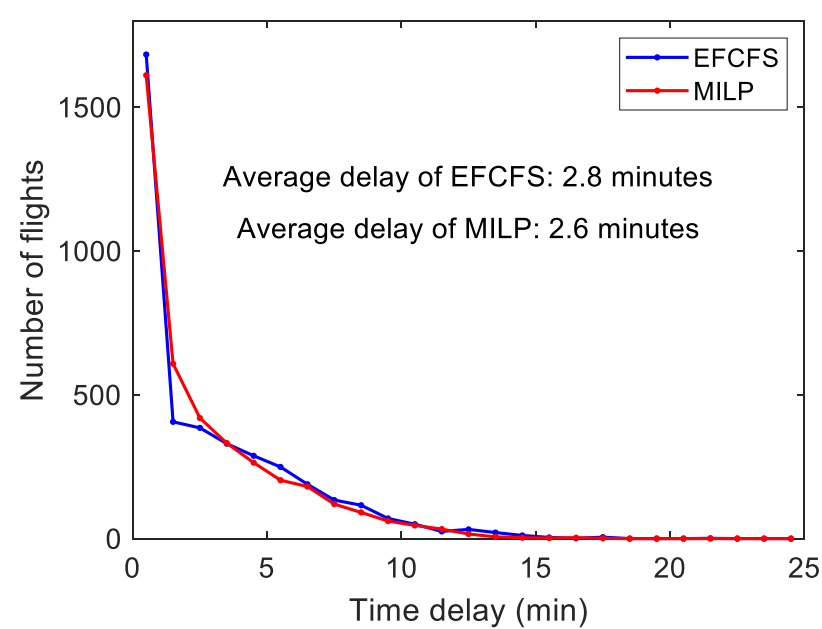

Gate departure delay

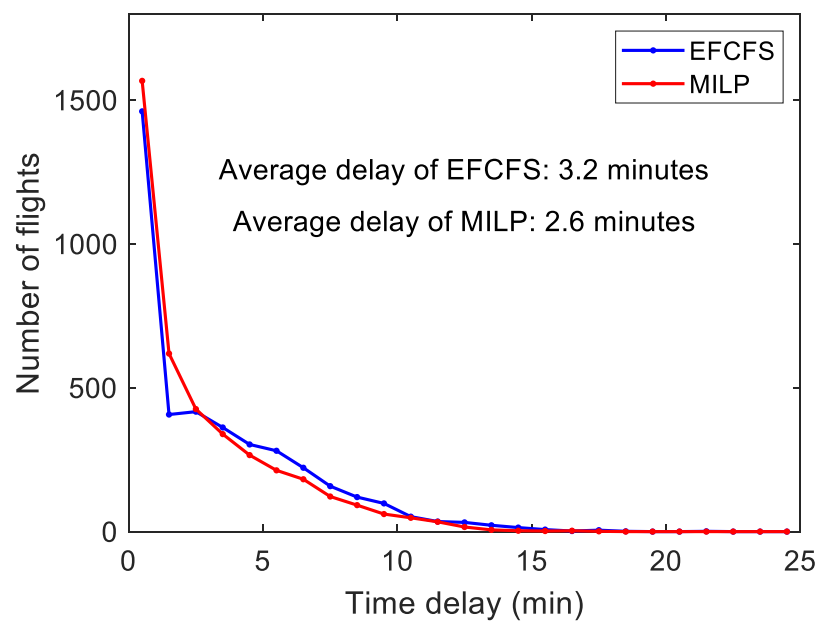

Runway takeoff delay 


\section{Scheduling Results - Case 1}

$>$ Maximum delay distributions

- MILP has better performances than EFCFS

- EFCFS is slightly shifted to the right side
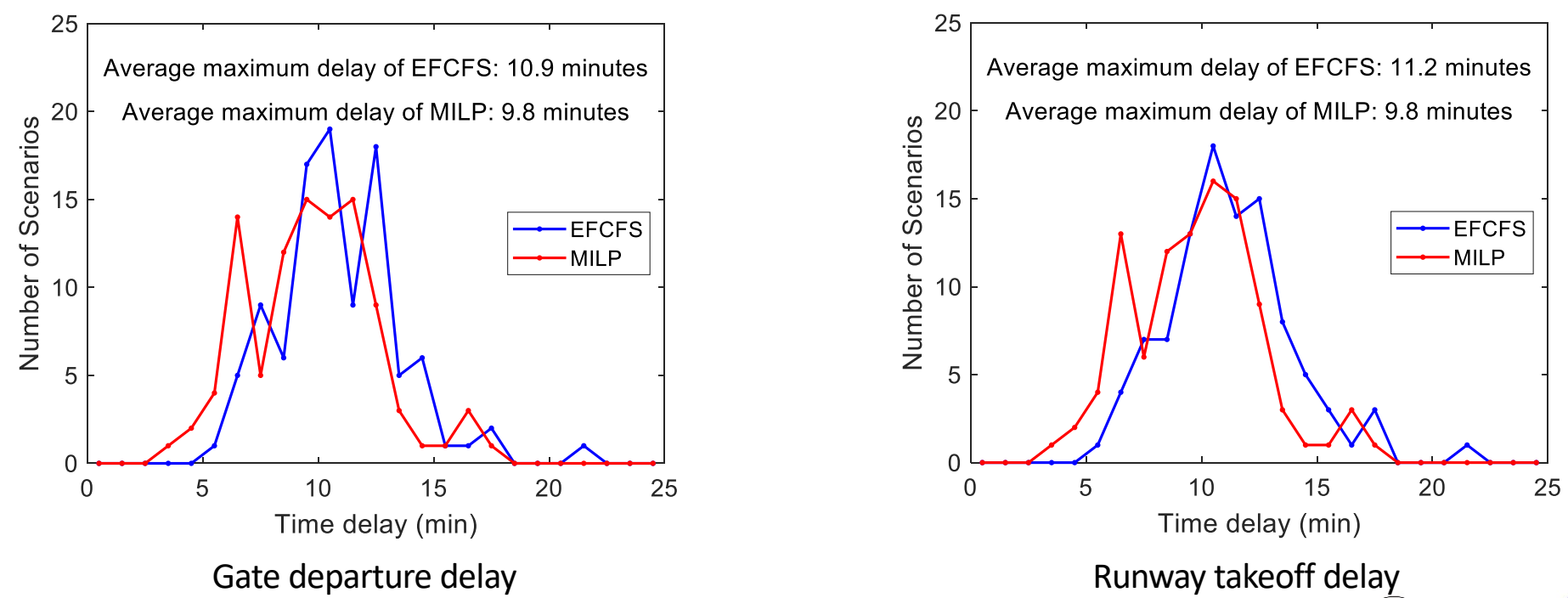

Runway takeoff delay 


\section{Scheduling Results - Case 1}

\section{$>$ Distribution of makespan differences}

- MILP shows slightly better performance

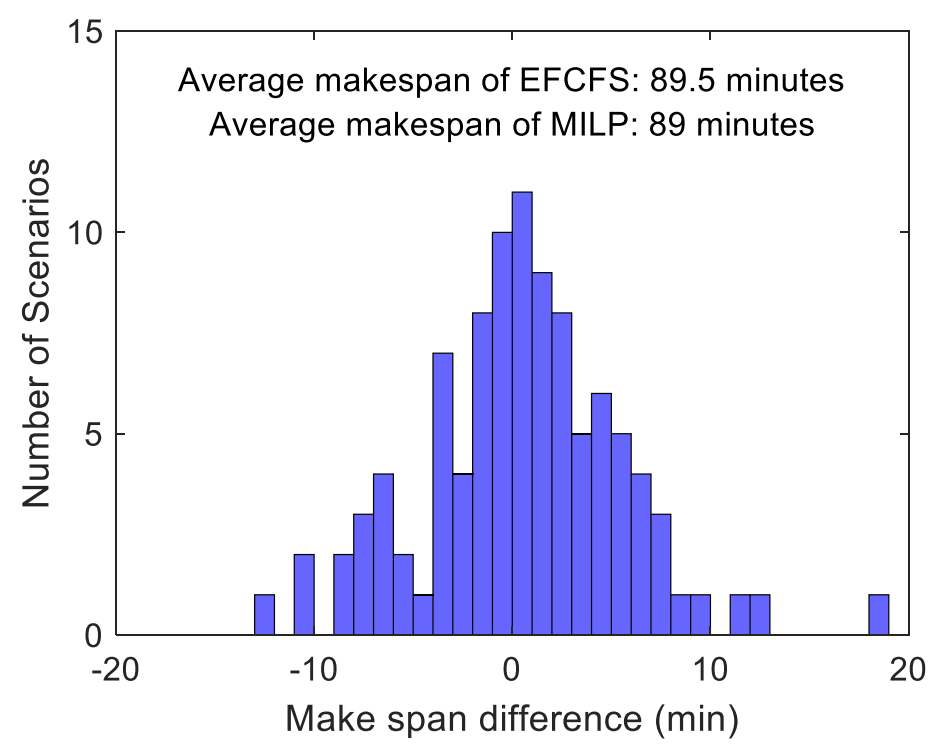




\section{Scheduling Results - Case 2}

\section{$>$ Delay distributions}

- EFCFS has more flights with 5-10 minutes runway takeoff delays

- MILP has more flights with the runway takeoff delays in 1 minute
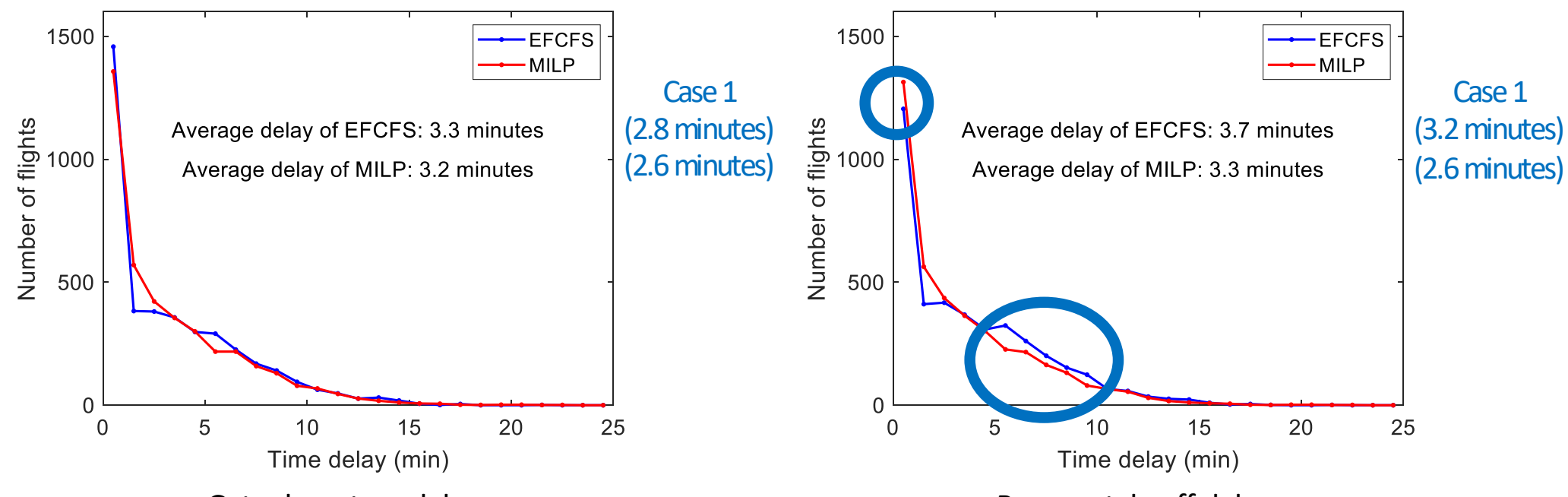

Gate departure delay

Runway takeoff delay 


\section{Scheduling Results - Case 2}

\section{$>$ Maximum delay distributions}

- The difference between MILP and EFCFS became smaller than Case 1

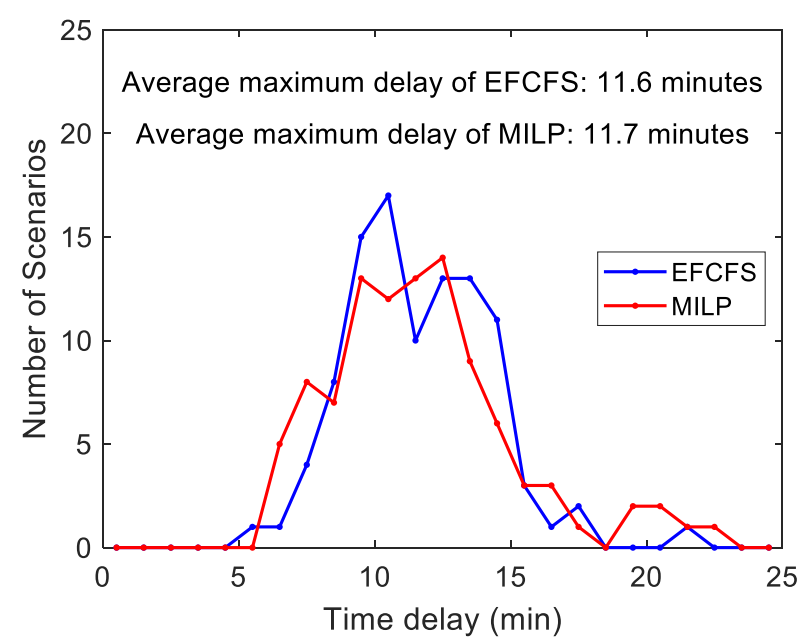

Gate departure delay

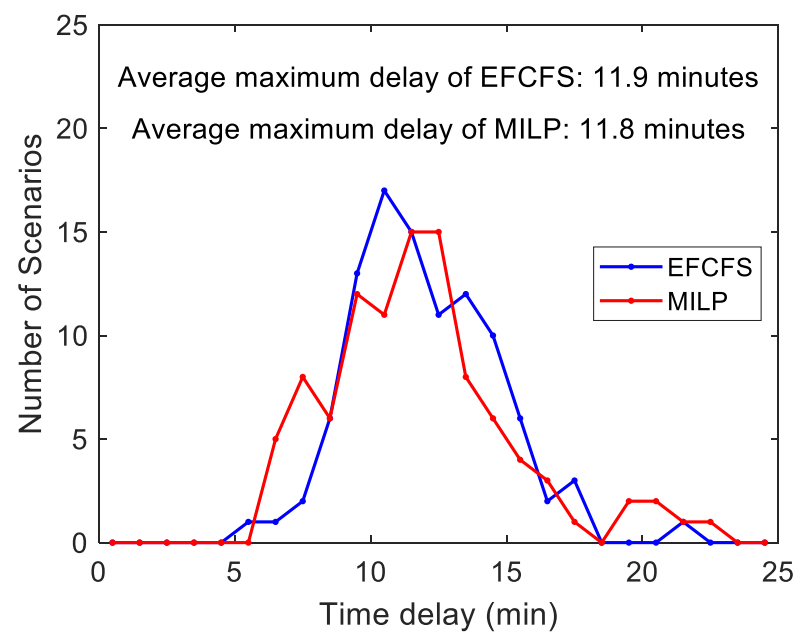

Runway takeoff delay 


\section{Scheduling Results - Case 2}

$>$ Distribution of makespan differences

- MILP shows slightly better performance

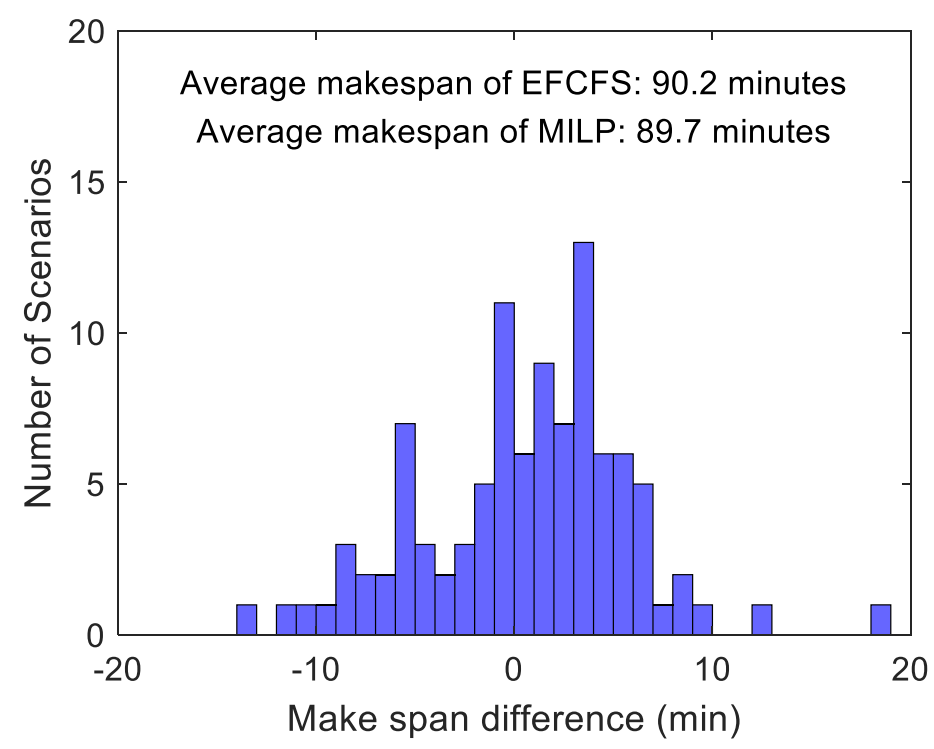




\section{Scheduling Results - Case 3}

\section{$>$ Delay distributions}

- EFCFS shows larger average delays for both gate departure and runway takeoff

- EFCFS has more flights with the delays larger than 70 minutes

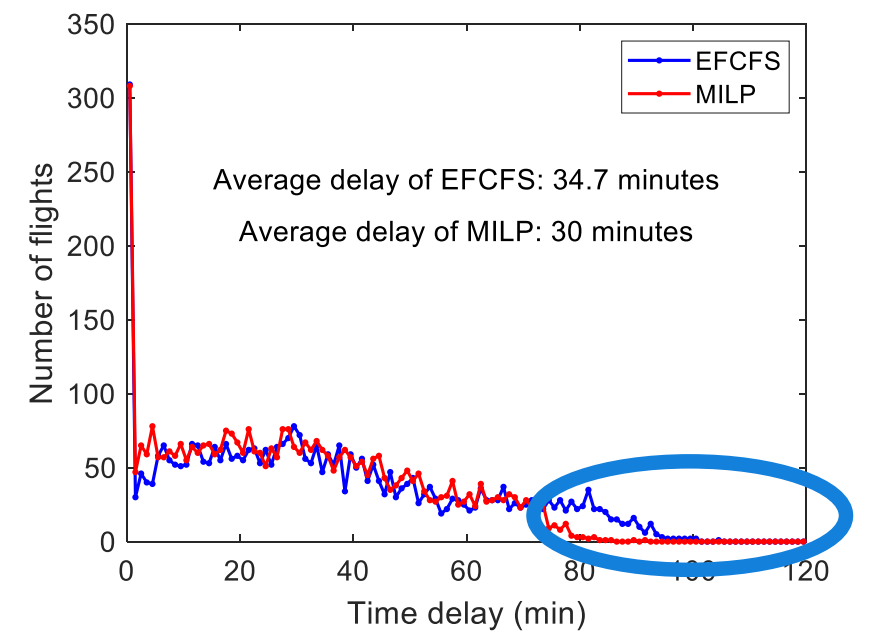

Gate departure delay

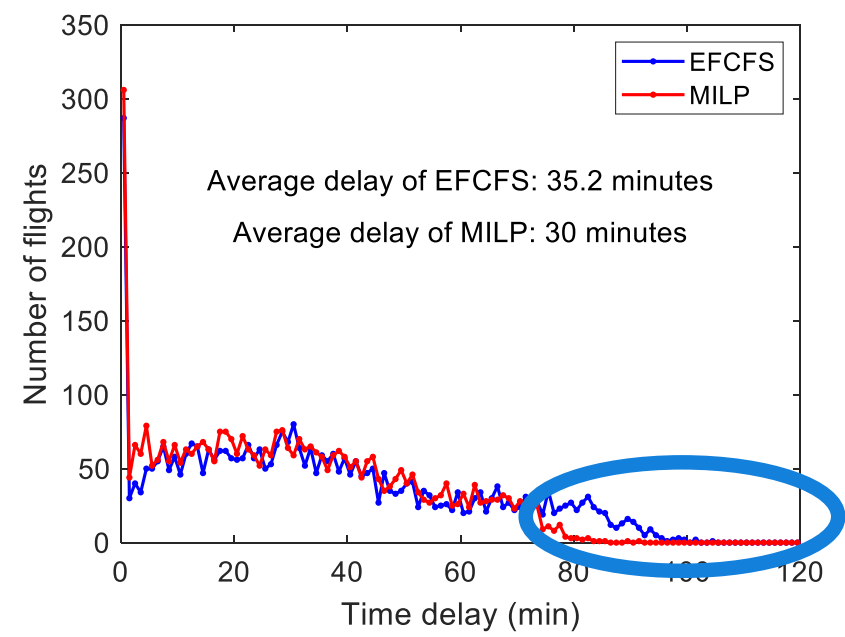

Runway takeoff delay 


\section{Scheduling Results - Case 3}

$>$ Maximum delay distributions

- EFCFS produced larger maximum delays

- Distributions of EFCFS are shifted to the right side

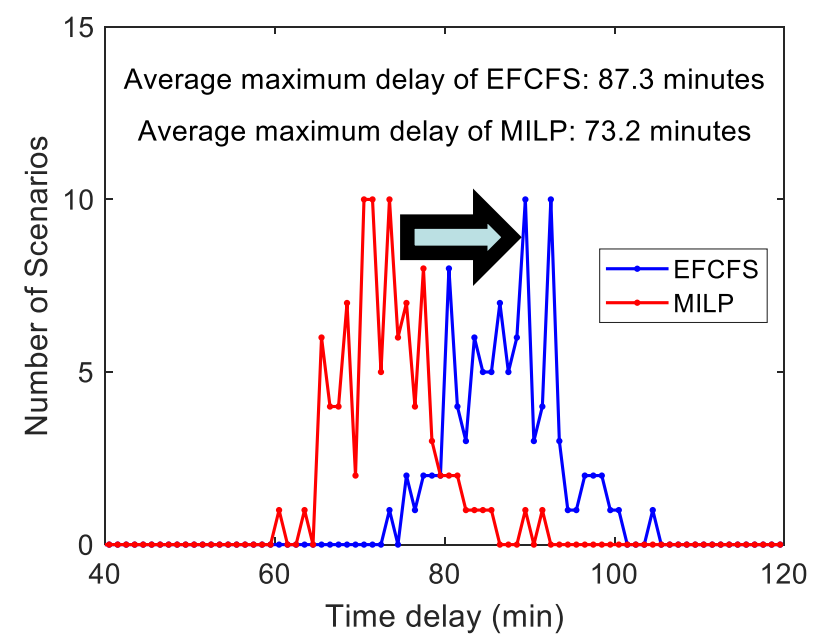

Gate departure delay

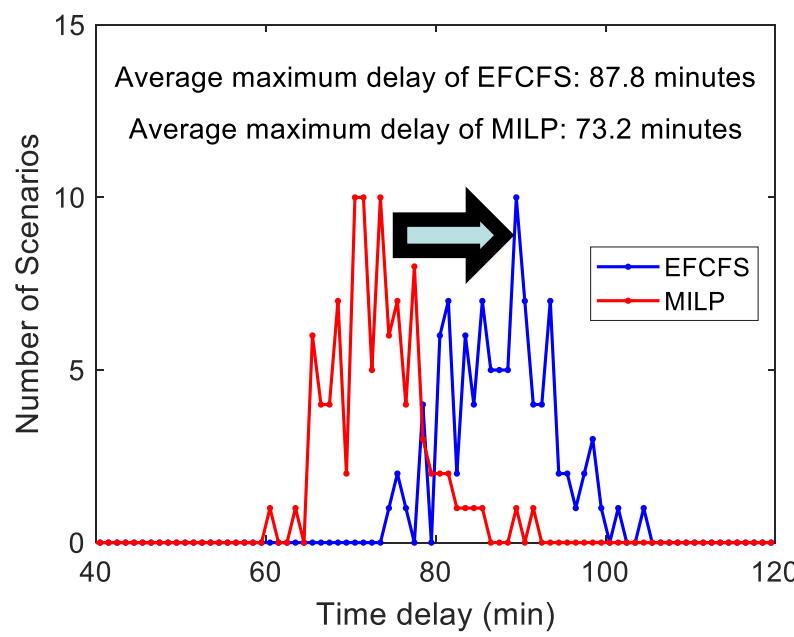

Runway takeoff delay 


\section{Scheduling Results - Case 3}

\section{$>$ Distribution of makespan differences}

- The Makespan differences are biased in the positive direction

- MILP shows much better performance with large runway separations

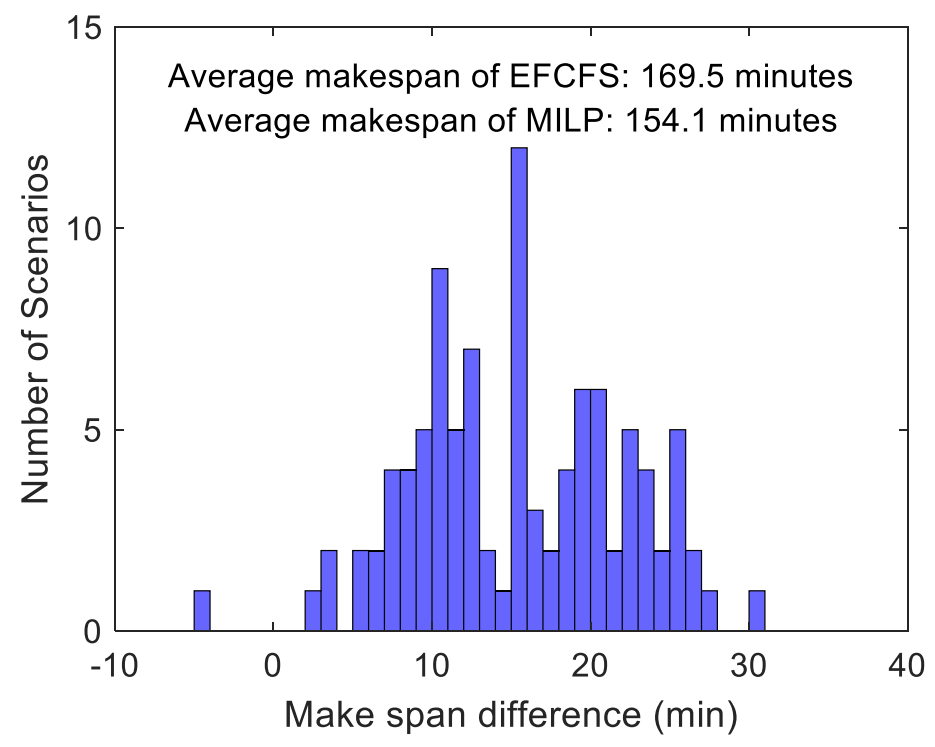




\section{Computation Times}

\section{Case 1 (No MIT)}

- EFCFS: 0.82 seconds

- MILP: 6.39 seconds

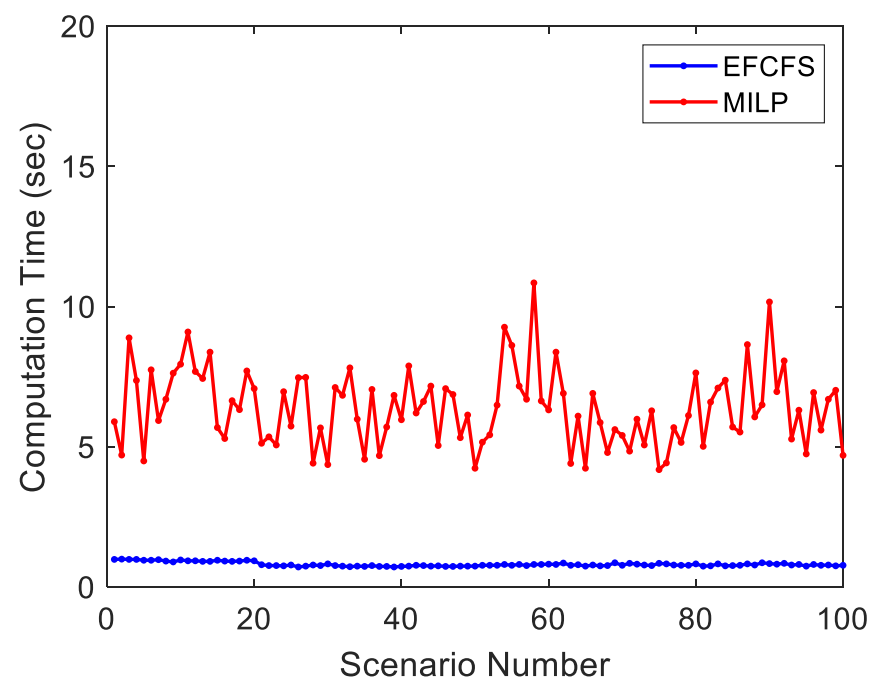

* Desktop specification

Intel i7-6820HQ, $2.79 \mathrm{GHz} / 32 \mathrm{~GB}$ RAM
Case 2 (with MIT)

- EFCFS: 0.99 seconds

- MILP: 9.22 seconds

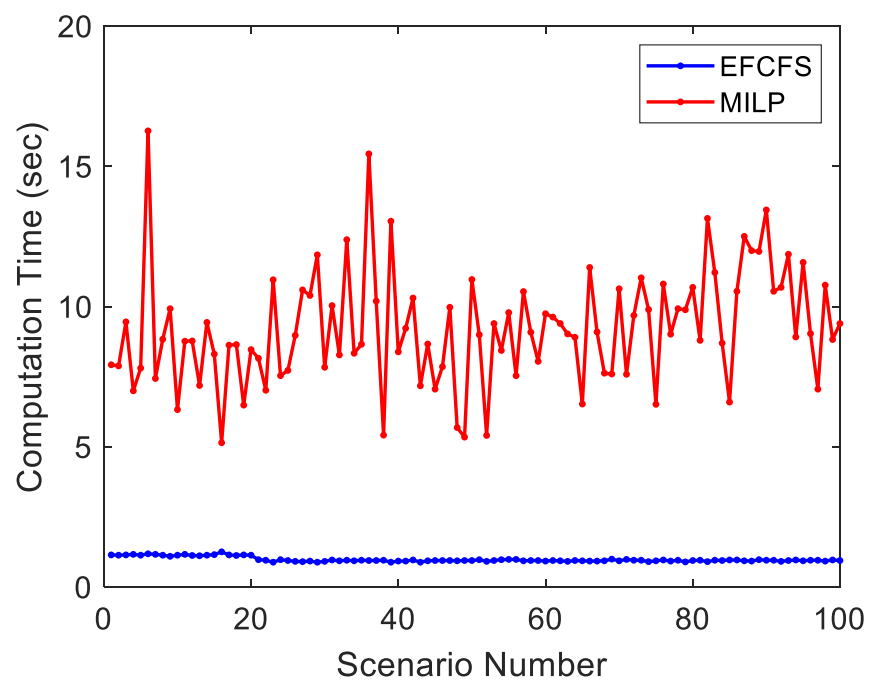




\section{Scheduling results - Summary}

\section{$>$ MILP}

- Slightly smaller average and maximum takeoff delays

- Slightly smaller average makespans

$>$ EFCFS is about 10 times faster for the given problem size.

$>$ MILP's advantage is more noticeable in high delay situations.

$>$ Applying MIT constraints

- The differences in results between EFCFS and MILP became smaller.

- The computation times of MILP were increased. 


\section{CONCLUSIONS}




\section{Conclusions}

$>$ Two different scheduling approaches were compared

- Common constraints were considered

- 100 scenarios were randomly generated

$>$ MILP generally showed better performance in terms of minimizing delays, but the differences were small.

$>$ EFCFS is much faster in computational performance

- Real time situations

- Scheduling large number of aircraft 


\section{Future Research Plans}

$>$ Testing more scenarios considering higher delay such as operations with severe weather condition or future traffic demand

$>$ Handling uncertainty

- Add buffer times

- Update periodically with fast-time simulation

- Use probabilistic model 


\section{Questions?}

